\title{
COVID-19 was found in A Patient's CSF Who Presented with A Severe form of Guillain Barre Syndrome; A Successful Sudanese Story: A Case Report
}

Etedal Ahmed A. Ibrahim

Al Neelain University, Faculty of Medicine

Khabab Abbasher Hussien Mohamed Ahmed ( $\sim$ Khabab9722@gmail.com )

University of Khartoum, Faculty of Medicine https://orcid.org/0000-0003-4608-5321

Elmuntasir Taha Salah

National Ribat University, Faculty of Medicine

\section{Case Report}

Keywords: COVID-19, Guillain-Barre syndrome, Neurology

Posted Date: June 14th, 2021

DOI: https://doi.org/10.21203/rs.3.rs-616410/v1

License: (c) (i) This work is licensed under a Creative Commons Attribution 4.0 International License.

Read Full License 


\section{Abstract \\ Background:}

Neurological manifestation and complications are common due to the coronavirus infectious disease COVID-19. It affects higher functions, cranial nerves and the motor system. It can lead to headaches, convulsions, mental and psychological changes like delirium and insomnia. Guillain Barre syndrome rarely occurs as a consequence of or in co-incidence with COVID-19. The authors report a case of Guillain Barre syndrome as an example of a success story in managing a complicated case of COVID-19 in an elderly male with signs of a poor prognosis.

\section{Case presentation:}

A previously healthy 70-year-old man presented (on the 25th of June, 2020) with a fever and cough followed by quadriplegia and facial weakness one week later. He tested positive for COVID-19, and a nerve conduction study revealed demyelinating neuropathy consistent with Guillain Barre syndrome. He received treatment in the form of intravenous immunoglobulin with marked improvement despite poor prognostic features.

\section{Conclusion:}

Patients with COVID-19 can present with any symptoms, including diseases of the nervous system and peripheral nerves such as Guillain Barre syndrome, which respond very well to IVIG treatment despite poor prognostic factors such as old age, gender, rapid onset of complete paralysis, lymphopenia and a groundglass appearance on CT chest scans, which all existed in this case.

\section{Background:}

The World Health Organization (WHO) was notified in December 2019 about COVID-19, a new coronavirus detected in Wuhan, China, as the cause of an outbreak of a lower respiratory tract infection. ${ }^{1}$ The WHO then declared it a Public Health Emergency of International Concern on 30 January 2020. On 11 February 2020, the WHO announced a name for the new coronavirus disease: COVID-19. ${ }^{2}$ To date, there are 8,860,331 cases of COVID-19 globally including 465,740 deaths. The highest number of cases are in America at 4,370,519 with 4,996 deaths. In the Eastern Mediterranean WHO region, there are 914,518 cases with 20,531 deaths. Sudan is a member of the Eastern Mediterranean WHO region and reported 8,580 with 521 deaths. $^{3}$ The virus mainly causes pneumonia and acute respiratory distress syndrome ${ }^{4}$, as well as a multi-organ disease affecting the kidneys, brain, heart, liver and other organs. ${ }^{5}$ It leads to serious complications such as a cytokine storm, septic shock, blood clots and immune-mediated injuries. ${ }^{6-8}$ Neurological manifestations and complications are common due to COVID-19. It affects higher functions, cranial nerves and the motor system. It can lead to headaches, convulsions, mental and 
psychological changes, delirium and insomnia. Guillain Barre syndrome can occur as a consequence of

or in co-incidence with COVID-19, but it is very rare. ${ }^{9-11}$ The authors report a case of Guillain Barre syndrome as an example of a success story in managing a complicated case of COVID-19 in an elderly male with signs of a poor prognosis.

\section{Case Presentation:}

A previously healthy 70-year-old male (without hypertension or diabetes) presented (on the 25th of June, 2020) at the Emergency Department with complaints of lower limb weakness with an acute onset of numbness and the feeling of dead lower limbs preceded by a cough, which was dry and paroxysmal, accompanied by mild chest discomfort and a high-grade fever without sweating or rigours. The fever and cough lasted for 7 days before the occurrence of weakness. His condition progressed over a day involving the upper limbs, neck and facial muscles, and the patient was unable to turn in bed, stand, walk independently, move his upper limbs or close his eyes. Difficulty swallowing, nasal regurgitation or choking was not seen, and he had normal sensations and sphincters. Additionally, no convulsions, loss of consciousness or other symptoms related to cranial nerves or higher functions were seen.

On examination, the patient was conscious, alert, and orientated to time, place and person. A mini-mental status examination (MMSE) was at 30. A cranial nerves examination revealed bilateral facial nerve palsy on the right side with facial deviation to the left, and the inability to close both eyes and blow his cheeks to whistle. Nystagmus, ophthalmoplegia, diplopia, cerebellar symptoms and bulbar palsy were not detected. He had a normal jaw jerk with weak neck flexion. Furthermore, an upper limbs examination showed hypotonia with absent reflexes and a muscle power assessment (MRC) was at grade 3 proximally and grade 2 distally, with normal sensations and absent tendon reflexes. A lower limbs examination also revealed hypotonia with an MRC of grade 2 proximally and distally, absent reflexes, normal sensations, a flexor plantar response with normal coordination, and the patient was unable to walk.

General investigations were conducted with complete blood counts showing haemoglobin $(\mathrm{Hb}) 11 \mathrm{~g}$, total white blood cells 6 , lymphocytes $12 \%$, C-reactive protein (CRP) 110, erythrocyte sedimentation rate 70 , platelets 396 , serum ferritin $1000 \mathrm{ng} / \mathrm{ml}$, blood urea $40 \mathrm{mg} / \mathrm{dL}$, serum creatinine $0.9 \mathrm{mg} / \mathrm{dL}$, serum potassium $3.5 \mathrm{mmol} / \mathrm{L}$, sodium $135 \mathrm{mmol} / \mathrm{L}$, alanine transferase 40 , aspartate transaminase 20, alkaline phosphatase 150, random blood sugar $120 \mathrm{mg} / \mathrm{dL}$, and a positive COVID-19 test. A computerized tomography (CT) chest scan showed a ground-glass appearance (Fig. 1\&2), and a nerve conduction study (NCS) reported demyelinating neuropathy consistent with acute inflammatory demyelinating polyradiculoneuropathy. Although it is a rare finding, a cerebrospinal fluid (CSF) examination was positive for both COVID-19 \& supporting the diagnosis of Guillain-Barre syndrome.

\section{Discussion:}


Acute inflammatory demyelinating polyradiculoneuropathy or Guillain Barre syndrome is an immunemediated nerve disease. Reported causes of the syndrome are campylobacter, mycoplasma, influenza, Zika virus, cytomegalovirus, HIV and lymphoma. ${ }^{12}$ Coronavirus (SARS-COV 2) or COVID-19 is a rare cause of Guillain Barre syndrome. ${ }^{11-13}$ There are very few cases worldwide with COVID-19 causing GBS with some of these cases showing a good response to intravenous immunoglobulin. ${ }^{14}$ Other cases showed axonal neuropathy in the NCS, while others showed demyelinating neuropathy which is a common type in North America and Europe but thought to be rare in Africa. Other types of GBS according to the NCS classifications are acute motor axonal neuropathy (AMAN) and acute sensory motor neuropathy (ASMAN) which are more frequent in China, Japan and Mexico, and Miller Fisher syndrome (MFS) which is more common in Asia. ${ }^{15}$ In Sudan, we have mixed types of AIDP, AMAN, ASMAN and MFS. ${ }^{16}$ In this case, the patient first presented with weakness ascending in nature involving the upper limbs, neck and facial muscles on the same day, preceded by a high-grade fever with rigor and sweating, a dry cough, soreness, and chest discomfort with normal sensations, sphincter and flexor plantar responses. The patient came to the Emergency Department at the National Centre for Neurological Sciences in Khartoum with signs suggestive of COVID-19 infection-causing GBS. A patient workup was conducted including general investigations and complete blood counts which showed lymphopenia, high CRP and serum ferritin levels, normal arterial blood gases and the presence of a ground-glass appearance which is highly suggestive of COVID-19 in conjunction with the symptoms. A nasal swab was taken and sent to the lab. After that, treatment with intravenous immunoglobulin was started in doses of $28 \mathrm{~g}$ per day. While the nasal swab result was pending, the patient showed immediate improvement after IVIG; the power changed from MRC grade 3 to MRC grade 2. Moreover, the patient received supportive management for COVID-19 in the form of paracetamol and vitamins. The patient reported that he was satisfied with the outstanding response to the treatment. A nerve conduction study showed a decrease in conduction velocity and delayed latencies with a dispersed response. This was due to the presence of demyelination, which is suggestive of the diagnosis of acute inflammatory demyelinating polyradiculoneuropathy or GBS. A follow-up with the patient after one month showed complete recovery, the patient walking without support.

\section{Conclusion:}

Patients with COVID-19 can present with any symptoms, including diseases of the nervous system and peripheral nerves such as Guillain Barre syndrome, which sometimes responds to IVIG treatment. In this case, there was an excellent response despite the poor prognostic factors such as old age, gender, rapid onset of complete paralysis, lymphopenia, high inflammatory markers, and a ground-glass appearance on a CT chest scan. The presence of the virus can be seen in the CSF, which existed in this case.

\section{Abbreviations}

AIDP

Acute inflammatory demyelinating polyradiculoneuropathy 
AMAN

Acute motor axonal neuropathy

ASMAN

Acute sensory motor axonal neuropathy

COVID-19

coronavirus disease of 2019

CNS

Central nervous system

CRP

C-reactive protein

CSF

Cerebrospinal fluid

CT

Computerized tomography

GBS

Guillain Barre syndrome

IVIG

Intravenous immunoglobulin

MERS

Middle East respiratory syndrome

MFS

Miller Fisher syndrome

MMSE

Mini-mental status examination

MRC

Muscle power assessment

NCS

Nerve conduction study

SARS

Severe acute respiratory syndrome

SARS-CO2

Severe acute respiratory syndrome coronavirus 2

WHO

World Health Organization

\section{Declarations}

\section{Ethics approval:}

Not applicable 


\section{Consent to participate:}

Verbal and written consents were obtained from the patient before writing the case or using investigations.

\section{Consent for publication:}

Written consent to publish this information was obtained from the patient. The patient gave written consent for his personal clinical details along with his CT chest images to be published in this study. This patient has not been reported in any other submission by the authors or anyone else.

\section{Availability of data and materials:}

The datasets used and/or analysed during the current study are available from the corresponding author on reasonable request.

\section{Competing interests:}

The authors have no conflict of interest to declare.

\section{Funding:}

The study was funded by the authors themselves.

\section{Authors' contributions:}

EIA: The first author collected the data, analysed the results and wrote the manuscript. KH: The second author wrote the manuscript, revised the manuscript and did editing. EST: The third author collected and analysed the data. All authors read and approved the final manuscript.

\section{Acknowledgement:}


We would like to acknowledge the National Centre for Neurological Sciences and the Neurology Department for their support and help.

\section{Authors' information:}

1. Alneelain University, Khartoum, Sudan 2. The National Centre for Neurological Sciences, Khartoum, Sudan

3. Medical student, University of Khartoum, Faculty of Medicine, Khartoum, Sudan,

4. National Ribat University Khartoum, Sudan.

\section{ORCID ID:}

Khabab Abbasher https://orcid.org/0000-0003-4608-5321

\section{References}

1. Christian N, Nadia H, Fidele B, Nsanzimana S, Souopgui J, Leon M. The coronavirus disease 2019 (COVID-19) -A global health emergency.

2. World Health Organization Coronavirus disease (COVID-19) pandemic. https://www.who.int/emergencies/diseases/novel-coronavirus-2019

3. World Health Organization. (2020). Coronavirus disease 2019 (COVID-19): situation report, 154. World Health Organization.

4. Sardar R, Satish D, Birla S, Gupta D. Comparative analyses of SAR-CoV2 genomes from different geographical locations and other coronavirus family genomes reveals unique features potentially consequential to host-virus interaction and pathogenesis. BioRxiv. 2020 Jan 1.

5. Chowdhury SD, Oommen AM. Epidemiology of COVID-19. Journal of Digestive Endoscopy. 2020 Mar;11(01):03-7.

6. Chen N, Zhou M, Dong X, Qu J, Gong F, Han Y, Qiu Y, Wang J, Liu Y, Wei Y, Yu T. Epidemiological and clinical characteristics of 99 cases of 2019 novel coronavirus pneumonia in Wuhan, China: a descriptive study. The Lancet. 2020 Feb 15;395(10223):507-13.

7. Ye Q, Wang B, Mao J. The pathogenesis and treatment of the Cytokine Storm in COVID-19. The Journal of infection. 2020 Jun.

8. Wu Y, Xu X, Chen Z, Duan J, Hashimoto K, Yang L, Liu C, Yang C. Nervous system involvement after infection with COVID-19 and other coronaviruses. Brain, behaviour, and immunity. 2020 Mar 30. 
9. Sedaghat Z, Karimi N. Guillain Barre syndrome associated with COVID-19 infection: a case report. Journal of Clinical Neuroscience. 2020 Apr 15.

10. Dalakas MC. Guillain-Barré syndrome: The first documented COVID-19-triggered autoimmune neurologic disease: More to come with myositis in the offing. Neurology-Neuroimmunology Neuroinflammation. 2020 Sep 1;7(5).

11. Zhao H, Shen D, Zhou H, Liu J, Chen S. Guillain-Barré syndrome associated with SARS-CoV-2 infection: causality or coincidence? The Lancet Neurology. 2020 May 1;19(5):383-4.

12. Krauer F, Riesen M, Reveiz L, Oladapo OT, Martínez-Vega R, Porgo TV, Haefliger A, Broutet NJ, Low N, WHO Zika Causality Working Group. Zika virus infection as a cause of congenital brain abnormalities and Guillain-Barré syndrome: systematic review. PLoS medicine. 2017 Jan 3;14(1):e1002203.

13. Willison HJ, Jacobs BC, Van Doorn PA. Guillain-barre syndrome. The Lancet. 2016 Aug 13;388 (10045):717-27.

14. Van Den Berg B, Walgaard C, Drenthen J, Fokke C, Jacobs BC, Van Doorn PA. Guillain-Barré syndrome: pathogenesis, diagnosis, treatment and prognosis. Nature Reviews Neurology. 2014 Aug;10(8):469-82.

15. Wakerley BR, Kokubun N, Funakoshi K, Nagashima T, Hirata K, Yuki N. Clinical classification of 103 Japanese patients with Guillain-Barre syndrome. Journal of the neurological sciences. 2016 Oct 15;369:43-7.

16. Ibrahim EAA; Guillain Barre syndrome in Sudan. Under press.

\section{Figures}




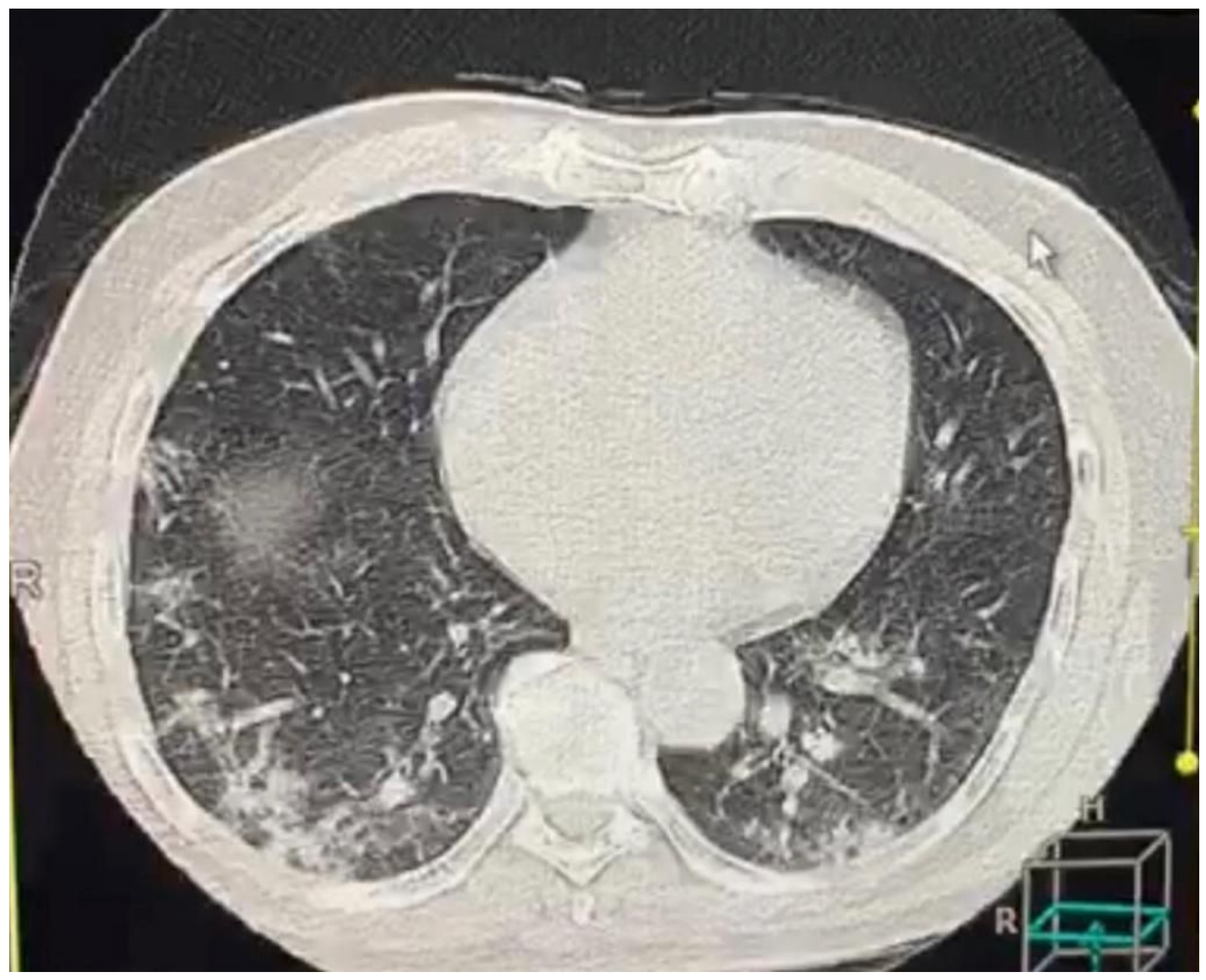

Figure 1

Computerized tomography of the chest of a 70-year-old male showing a ground-glass appearance 


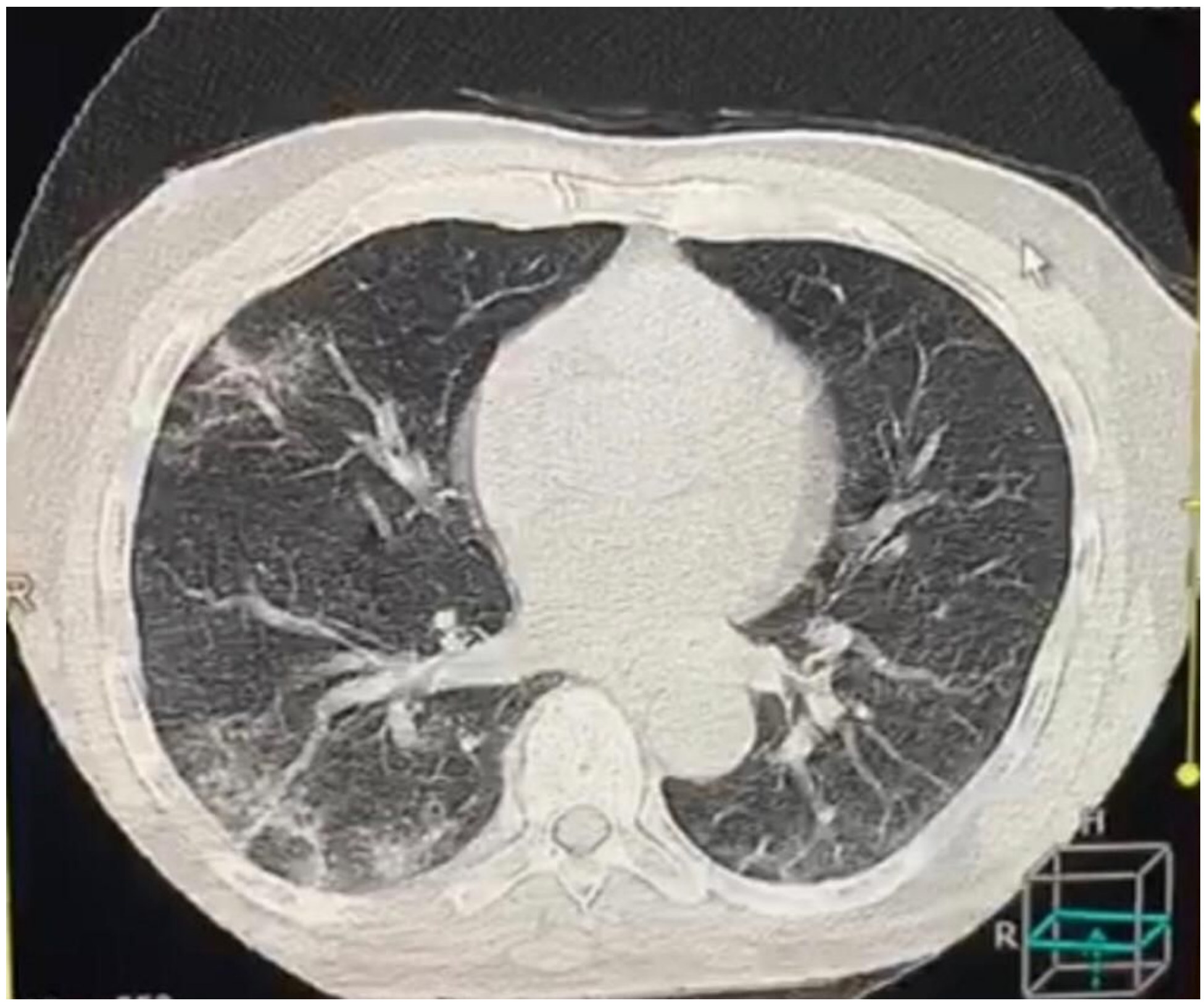

Figure 2

Computerized tomography of the chest of a 70-year-old male showing a ground-glass appearance

\section{Supplementary Files}

This is a list of supplementary files associated with this preprint. Click to download.

- IMG20210610WA0001.jpg

- IMG20210610WA0002.jpg 